\title{
Phase Transition Mapping by Means of Neutron Imaging in SOFC Anode Supports During Reduction Under Applied Stress
}

Makowska, Malgorzata; Strobl, M.; Lauridsen, E. M. ; Frandsen, Henrik Lund; Tremsin, Anton S.; Shinohara, T. ; Kuhn, Luise Theil

\author{
Published in: \\ E C S Transactions \\ Link to article, DOI: \\ 10.1149/06801.1103ecst \\ Publication date: \\ 2015 \\ Document Version \\ Peer reviewed version \\ Link back to DTU Orbit
}

Citation (APA):

Makowska, M., Strobl, M., Lauridsen, E. M., Frandsen, H. L., Tremsin, A. S., Shinohara, T., \& Kuhn, L. T. (2015). Phase Transition Mapping by Means of Neutron Imaging in SOFC Anode Supports During Reduction Under Applied Stress. E C S Transactions, 68(1), 1103-1114. https://doi.org/10.1149/06801.1103ecst

\section{General rights}

Copyright and moral rights for the publications made accessible in the public portal are retained by the authors and/or other copyright owners and it is a condition of accessing publications that users recognise and abide by the legal requirements associated with these rights.

- Users may download and print one copy of any publication from the public portal for the purpose of private study or research.

- You may not further distribute the material or use it for any profit-making activity or commercial gain

- You may freely distribute the URL identifying the publication in the public portal 


\title{
Phase transition mapping by means of neutron imaging in SOFC anode supports during reduction under applied stress.
}

\author{
M. G. Makowska ${ }^{\text {ab }}$, M. Strobl ${ }^{\text {b }}$, E. M. Lauridsen ${ }^{\text {c }}$, H. L. Frandsen ${ }^{\text {a }}$, A. S. Tremsin ${ }^{\text {d }}$,
} T. Shinohara ${ }^{\mathrm{e}}$ and L. Theil Kuhn ${ }^{\mathrm{a}}$

${ }^{a}$ Department of Energy Conversion and Storage, Technical University of Denmark, Frederiksborgvej 399, Roskilde, 4000, Denmark

${ }^{\mathrm{b}}$ European Spallation Source ESS AB, P.O Box 176, Lund, SE-221 00, Sweden

${ }^{c}$ Xnovo Technology ApS, Galoche Alle 15, Køge, 4600, Denmark

${ }^{\mathrm{d}}$ Space Sciences Laboratory, University of California at Berkeley, Berkeley, CA 9472

${ }^{\mathrm{e} J a p a n}$ Atomic Energy Agency, 2-4 Shirakata-shirane, Tokai-mura, Naka-gun, Ibarakiken 319-1195, Japan

Mechanical and electrochemical performance of layers composed of Ni-YSZ cermet in solid oxide fuel and electrolysis cells (SOC) depends on their microstructure and initial internal stresses. After sintering the manufacturing conditions, i.e. temperature, atmosphere and loads, can influence the microstructure and in particular the internal stresses in the Ni-YSZ layer and thereby the cell performance. Spatially resolved observation of the phase transition during reduction can provide information on how parameters like temperature and external load influence the reaction progress. This information is crucial for optimization of the SOC performance.

In this work the measurements with energy resolved neutron imaging of the phase transition during the NiO-YSZ reduction performed at different temperatures with and without applied load, are presented. The results indicate a link between reduction rate and stress, which will influence the initial stress field of the SOC stacks.

\section{Introduction}

Planar solid oxide electrochemical cells (SOC) are composed of at least three layers: an anode, an electrolyte and a cathode ${ }^{1-4}$. Cermet Ni-YSZ (yttria stabilized zirconia) is a material widely used in electrochemical devices like solid oxide electrolysis cells (SOEC) and solid oxide fuel cells (SOFC). It is used as electrochemically active anodes in SOFC and cathodes in SOEC, but also serves as a mechanical supports for the SOC. Mechanical and electrochemical performance of these layers, and thus whole cells, depends on the material microstructure ${ }^{5-9}$. Because of the differences in thermal expansion coefficients in the multiple layers of SOCs internal initial stresses will be present in the SOCs. Stress, which occurs due to the incompatibility of thermal expansion coefficients of layers leads to significant bending of SOC, unless they are pressed in the stack ${ }^{10,11}$.

One of the commonly used manufacturing techniques of the Ni-YSZ support layer is tape-casting from slurry containing NiO-YSZ particles, followed by sintering. Consequently the planar cells are stacked to achieve sufficient current density. In the stack the $\mathrm{NiO}$ is reduced to $\mathrm{Ni}$ during the initial preparation of the stack before operation. 
Conditions i.e. temperature, atmosphere and load applied during the stacking and reduction procedure, determine the microstructure and initial internal stress distribution of the support and thereby the cell performance. Therefore, investigation of the reduction process under different conditions can provide knowledge needed to optimize the SOC performance. Especially useful information can be achieved using spatially resolved measurement techniques enabling monitoring changes of the different phases distribution in time as the reduction procedure are not necessarily uniform across a SOC ${ }^{12,13}$.

Recently, two new phenomena linking stress field and reduction rates in anode supports for SOFCs have been demonstrated i.e. accelerated creep during reduction and reduction nucleation due to stress ${ }^{12,14}$. The accelerated creep is several orders faster than the creep experienced in the SOCs during operation, and should relax al stresses in the cell at the point of reduction. Thus, the stress field and thus the integrity of a SOC stack is heavily dependent on this reduction process. Furthermore, it has been shown that applying an external load to an SOFC anode support during reduction can affect the reaction kinetics.

Previously feasibility of studying of these important phenomena for SOC development by means of energy resolved neutron imaging has been presented ${ }^{12}$. This technique applied to polycrystalline granular and porous materials can provide spatially resolved information about crystal structure, crystalline phases, texture and strain fields ${ }^{15-24}$. It has been shown in ${ }^{12}$ that due to excellent neutron scattering properties of $\mathrm{Ni}$, neutron imaging holds a particularly great potential for characterization of nickel-based components of solid oxide cells and the feasibility of applying energy resolved neutron imaging to investigations of $\mathrm{Ni}$ and $\mathrm{NiO}$ phase distributions in anode supports for $\mathrm{SOFC}$ was demonstrated.

In this work we present the results of more detailed energy resolved time of flight (TOF) neutron imaging investigations of the Ni-YSZ cermet used for SOFC anode supports. We have obtained local information about reduction process of NiO-YSZ samples, which have been loaded during the reduction. These samples are compared to unloaded reference samples, which has been exposed to the same reduction procedure, to elucidate the effect of the loading during reduction. Moreover, parts with different stress concentration within particular samples with respect to reduction rate were compared.

\section{Experimental}

\section{Sample preparation}

The samples used in this study were prepared by hot pressing of several layers of 300 $\mu \mathrm{m}$ thick tape casted NiO-YSZ tape. After hot pressing, samples were sintered and cut mechanically to dimensions $20 \times 6 \times 0.9 \mathrm{~mm}^{3}$. A more detailed description of the sample preparation can be found in our previous paper ${ }^{12}$, which presented studies of samples prepared using the same setup and methodology.

After sintering, the samples were reduced at elevated temperatures in an atmosphere of $9 \% \mathrm{H}_{2}$ with $91 \%$ of $\mathrm{N}_{2}$. During the reduction the samples were placed horizontally in a fixture. One side of the sample was fixed in the holder and the other side of the sample was mechanically loaded vertically, applying a bending moment on the sample. The bending moment give rise to a linear variation of axial stresses over the height of the sample with maximum tension at the upper surface of the sample and maximum compression at the lower surface. The reduction process was interrupted after a certain time (different time for each sample) and providing a series of samples with different 
reduction degrees, i.e. with different concentrations of $\mathrm{NiO}$ and $\mathrm{Ni}$ phases, and under the same load. The setup for the reduction process, as well as the reduction interruption procedure, is presented in detail in ref. ${ }^{12}$.

Before and after the reduction process the weight of the samples was measured with an analytical balance with accuracy $0.01 \mathrm{mg}$. For each sample the reduction degree was calculated from the measured weight loss and the ratio of $\mathrm{NiO}$ to YSZ. Series of samples were reduced at $650{ }^{\circ} \mathrm{C}$ with different reduction times $(0-60 \mathrm{~min})$ and reduction degrees in the range $0 \%-92 \%$ (weight loss normalized to $100 \%$ ). For comparison, a few samples were reduced at $700{ }^{\circ} \mathrm{C}$ and several reference samples (without applied load, but otherwise under the same conditions) were prepared.

\section{$\underline{\text { Time of flight energy resolved neutron imaging }}$}

Energy resolved neutron imaging is a technique based on the measurement of neutron transmission through the sample depending on neutron energy/wavelength. Such experiments can be performed both at continuous and pulsed neutron sources. In the first case, where a polychromatic continuous neutron beam is generated, either specific neutron wavelengths are selected using monochromators and subsequently transmission for different wavelengths is measured ${ }^{25}$, or the beam is chopped to generate a pulsed beam ${ }^{26,27}$. Consequently, separate pulses of neutrons travel over a certain distance $L$ to the sample, like it is in case of a pulsed source. Neutrons with shorter wavelengths (higher velocity) reach the sample earlier than those with longer wavelengths (lower velocity). As a result, neutrons with different energies arrive at the sample and subsequently at the detector at different times. Using detectors able to record simultaneously position and arrival time for every detected neutron, the energy resolved spectrum of the transmitted beam can be acquired for each pixel in the image. This approach is called the time of flight (TOF) imaging method. More details about this imaging technique can be found in ${ }^{15,19,23,28,29}$.

For polycrystalline samples, sudden increases of transmission/ decreases of attenuation occur at certain neutron wavelengths $(\lambda)$ in the recorded spectra. These features of the spectrum, so called "Bragg edges", correspond to crystal lattice planes with spacing values $\left(d_{h k l}\right)$ present in the investigated polycrystalline material, which are responsible for scattering neutrons out of the transmitted beam $(2 \alpha)$ according to the Bragg's law:

$$
n \lambda=2 d_{h k l} \sin \alpha
$$

Thus, Bragg edge patterns recorded with spatial resolution, contain local information about elements, crystal structure and phases, texture or strain field in a sample ${ }^{15-24}$.

In this work we present the results of energy resolved TOF neutron imaging measurements performed at the pulsed neutron spallation source of J-PARC (Japan) using the NOBORU instrument ${ }^{30,31}$. By means of a microchannel plate (MCP) detector $16,32,33$ with a pixel size of $55 \mu \mathrm{m}$ and field of view $2,8 \times 2,8 \mathrm{~cm}^{2}$, a series of TOF resolved radiographs (fig. 1) in the wavelength range 1-5 $\AA$ were acquired. Although every single neutron pulse provides the whole spectrum, in order to obtain reasonable statistics of measurement, the signal from many pulses, produced with a frequency of $25 \mathrm{~Hz}$, has been accumulated. For the presented results the exposure times were in range 4-5 h. 


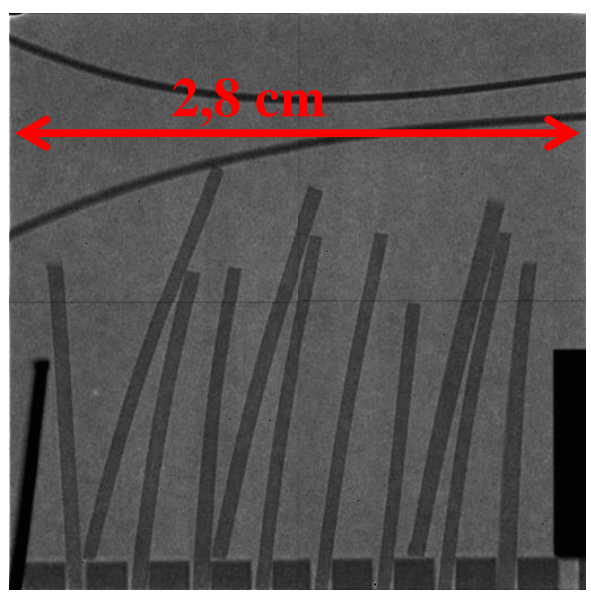

Fig. 1 Neutron radiograph with a set of SOFC anode support samples placed in the holder acquired for a certain neutron TOF, i.e. a defined wavelength, after $5 \mathrm{~h}$ of exposure.

Using the flight path of neutrons $L$, which was $14 \mathrm{~m}$, TOF values can be converted to neutron wavelengths for each acquired image, and thus the dependence of transmission on the neutron wavelength for any chosen part of the image can be evaluated. For normalization of the acquired data, the open beam was measured as well, and later images with samples were normalized by open beam images recorded for the corresponding TOF. The intrinsic detector time resolution was set to $10 \mu \mathrm{s}$, which translates into a wavelength resolution around $0.1 \%$ (depending on the wavelength) for the detection system. Since this value was significantly better than required, the recorded data were binned, during data processing, in order to obtain spectra with better statistics. For material characterization, it is more reasonable to consider the macroscopic cross section (i.e. the linear attenuation coefficient), which is independent of material thickness, instead of the directly measured transmission through the samples. For given beam transmission value and sample thickness, the macroscopic neutron cross section can be derived from the Lambert - Beer's law:

$$
\mu=\frac{1}{x} \ln \frac{I_{0}}{I},
$$

where $\mu$ is the macroscopic cross section, $\mathrm{x}$ is the sample thickness, and $I_{0}$ and $I$ are the intensities of incident and transmitted beam respectively.

\section{Results}

\section{$\underline{\text { Reduction degree vs time }}$}

The easiest way to define the reduction degree of the NiO-YSZ composite after a certain time under reducing conditions is to measure the weight of the sample before and after the process. This kind of measurement provides an integral reduction value for the whole sample volume and does not allow identification of local variations. However this is a very precise measurement. Figure 2 shows a plot of the dependence (for full samples) of the reduction degree calculated from sample weight loss measurement on reduction time. All the samples included in this plot were reduced at $650{ }^{\circ} \mathrm{C}$.

The phenomenological function fitted to the data points was:

$$
\frac{\Delta w}{\Delta w_{\infty}}=\left(1-e^{-\frac{t}{\tau}}\right),
$$


where $\Delta w$ is the weight loss (\% reduction) at time t, $\Delta w_{\infty}$ is the final weight loss, which was assumed to be equivalent to $100 \%$ reduction and $\tau$ is a characteristic time. The characteristic time was determined to be $17.2 \mathrm{~min}$.

It is apparent that the kinetics of the reaction gets slower at later stages of the process than predicted from the fitted function. This behavior is however expected, as different phenomena play a more significant role at later stages of the reduction of this material than at the beginning of the process. Among others, part of the $\mathrm{NiO}$ phase is difficult or even impossible to access for gas, so once the easily accessible $\mathrm{NiO}$ phase is already reduced, the rest undergoes reduction with much slower kinetics. Very similar dependencies were presented in ${ }^{34,35}$, however in these works reduction was conducted in atmosphere with lower hydrogen concentration, and therefore reaction was much slower.

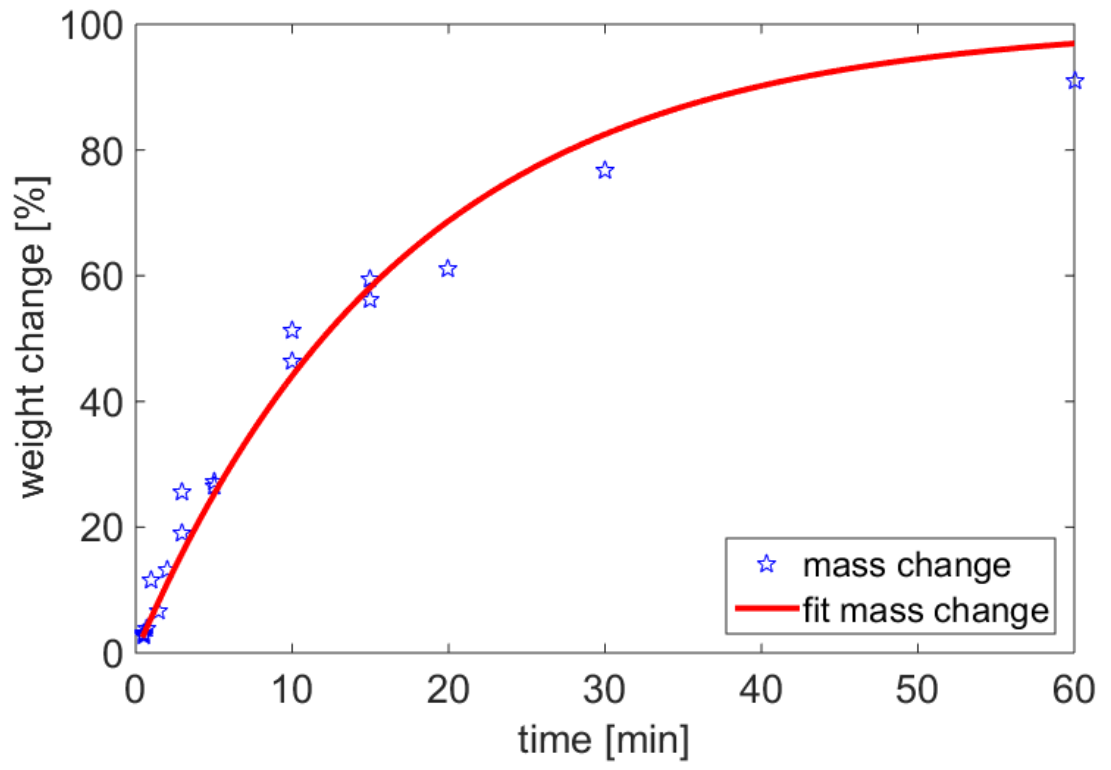

Fig.2 Normalized weight change against reduction time at $650{ }^{\circ} \mathrm{C}$.

\section{Mapping of reduction degree evaluated from Bragg edge patterns}

Figure 3 presents Bragg edge patterns for whole samples with known reduction degrees (calculated as a normalized weight loss during reduction process).

The Bragg edge patterns presented in fig. 3, which were evaluated for samples with different amounts of $\mathrm{NiO}$ and $\mathrm{Ni}$ phases, exhibit apparent differences for different reduction degrees. Achieving so well defined patterns with a relatively low noise level was possible due to long exposure time and a relatively large area selected for the signal averaging (full samples). As the overall reduction degrees for those samples are very well defined, here presented patterns can be used as a reference for quantification of the reduction degree for other samples or for selected parts of a particular sample. As presented in the following paragraph, this quantification can be done pixel-wise, resulting in a $2 \mathrm{D}$ mapping of the amount of $\mathrm{Ni}$ and $\mathrm{NiO}$ phases within the samples. The insert in the figure 3 presents examples of phase mapping for samples, which contained $11 \%$, $52 \%$ and $92 \%$ reduced $\mathrm{NiO}$ (i.e. $\mathrm{Ni}$ ), respectively. 


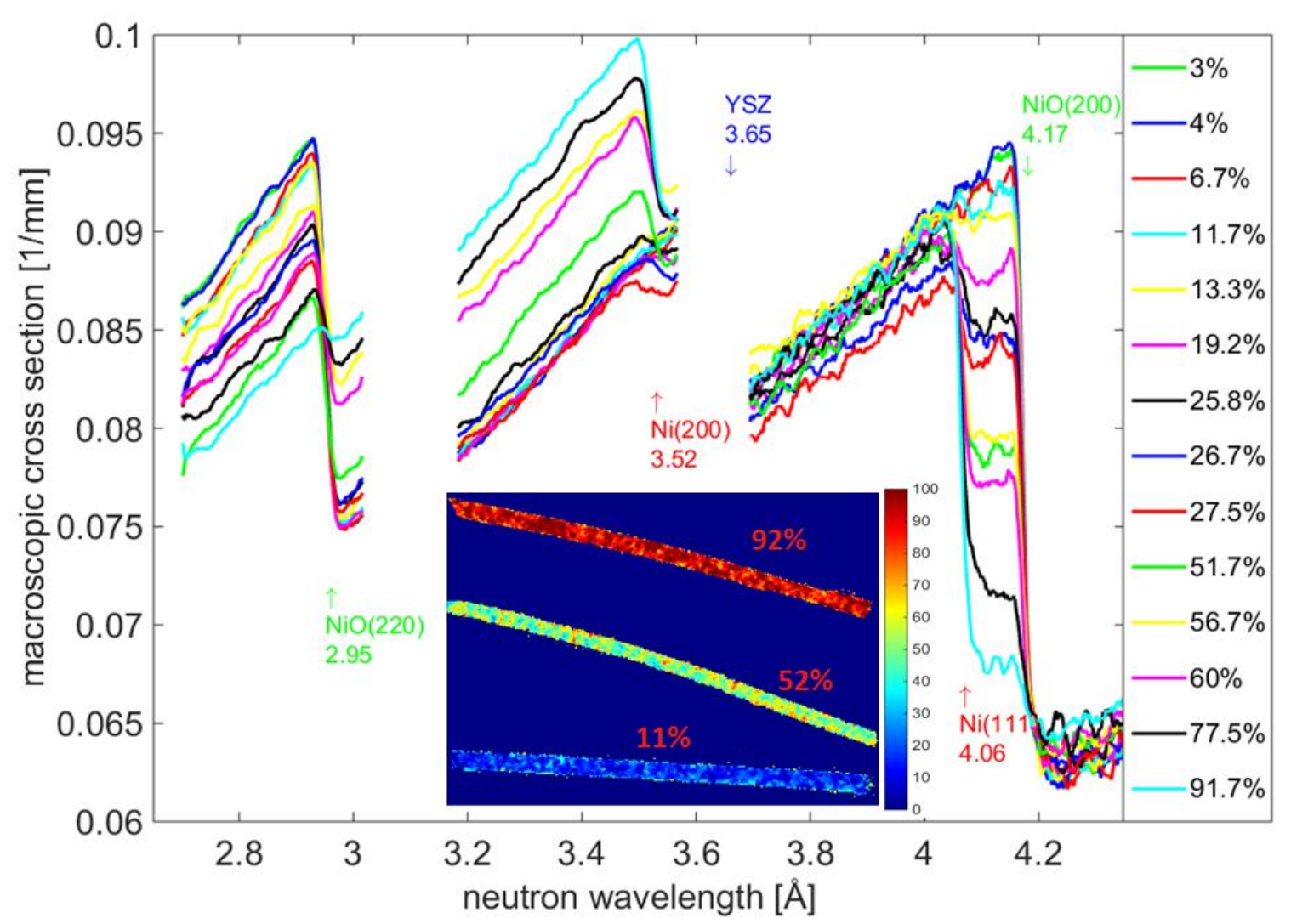

Fig. 3 Bragg edge patterns for full samples at different reduction stage with known percentage of reduced phase as indicated by the line color range shown to the right. The inset shows examples of pixel-wise quantified reduction degrees for samples containing an average of $11 \%, 52 \%$ and $92 \%$ of reduced phase in the full volume. The surface colors of the samples indicate the degree of reduction.

Quantification algorithm. For evaluation of reduction degree, four edges apparent in the patterns in fig.2a at $2.95 \AA, 3.52 \AA, 4.06 \AA$ and $4.17 \AA$, which correspond to the crystallographic planes $\mathrm{NiO}(220), \mathrm{Ni}(200), \mathrm{Ni}(111)$ and $\mathrm{NiO}(200)$ respectively, were taken into account. Although for shorter neutron wavelengths more edges can be observed, those four present in the part of the spectrum between $2.8 \AA$ and $4.2 \AA$ are significantly higher, thus including remaining edges in the algorithm would not improve the quantification.

According to the results presented in figure 3, an algorithm allowing for the calculation of the reduction degree from any evaluated Bragg edge pattern for the investigated material was derived. This quantification procedure is based on the following steps:

1. Analysis of the heights of Bragg edges appearing for wavelengths $2.95 \AA, 3.52 \AA$, $4.06 \AA$, 4.17 $\AA$, which correspond to crystallographic planes $\mathrm{NiO}(220), \mathrm{Ni}(200)$, $\mathrm{Ni}(111), \mathrm{NiO}(200)$ respectively.

2. The extracted edge heights vs. normalized weight change for different edges are plotted figure 4 . In case of edges at $4.06 \AA$ and $4.17 \AA$, only their ratio of their heights was plotted (fig. 4c), as due to very close localization, these two edges affect strongly each other. 
3. A linear relation between edge heights and weight change, i.e. reduction, as depicted in figure 4 is found. After such calibration:

4. Evaluation of Bragg edge pattern for certain region of interest in the image (e. g. part of sample) and evaluation of heights of different edges for this pattern.

5. Calculation of corresponding weight change values for the investigated individual areas/local patterns corresponding to the found linear relationships (Fig. 4).

6. Evaluation of the reduction degree for the considered part of image as an average of three values obtained in step 5 .

a)

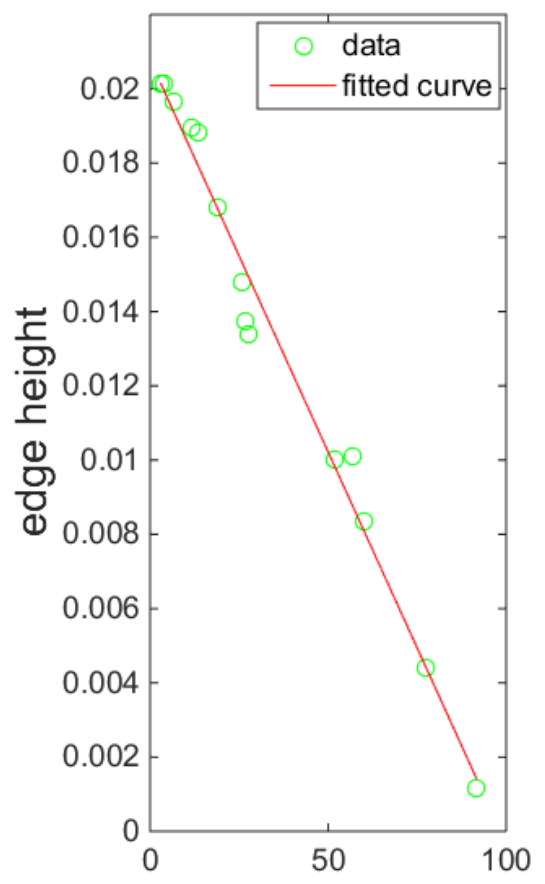

b)

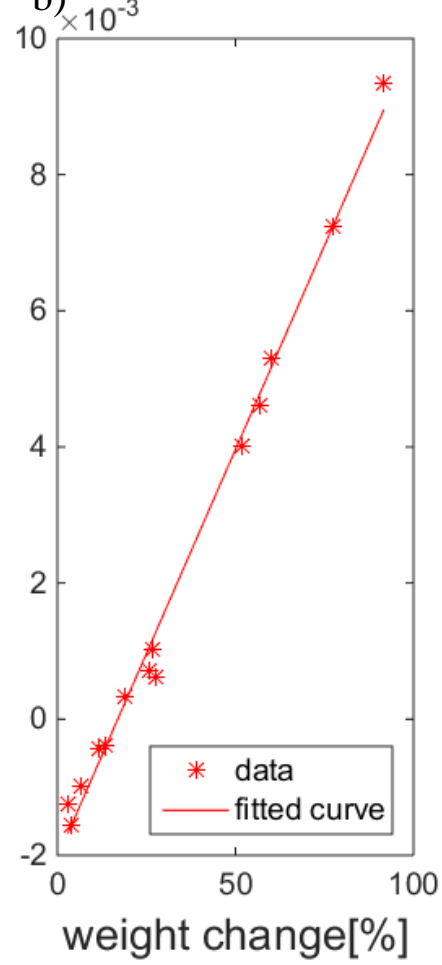

c)

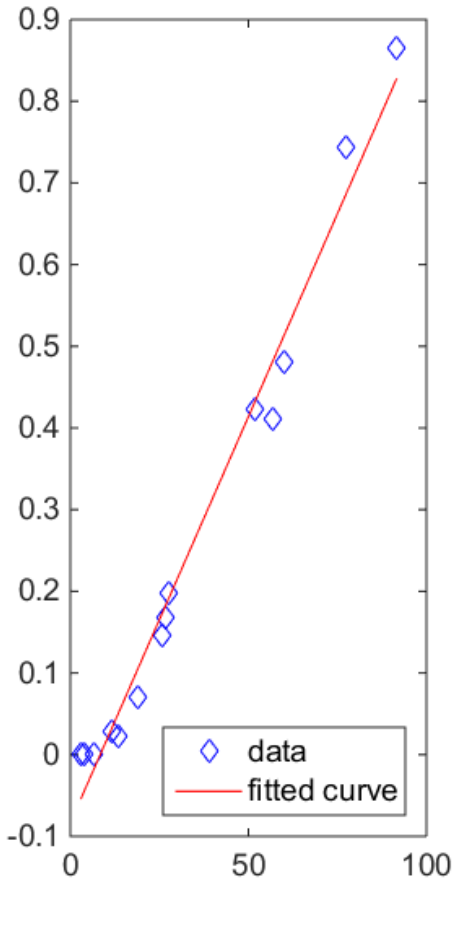

Fig.4 Dependence of Bragg edge heights on the sample weight change a) $\mathrm{NiO}(220)$ (2.95 $\AA$ ) edge height, b) $\mathrm{Ni}(200)(3.52 \AA)$ edge height, c) ratio of $\mathrm{Ni}(111)$ (4.06 $⿱$ $)$ and $\mathrm{NiO}(200)(4.17 \AA)$ edge heights.

\section{Effect of stress on reduction progress}

In this paragraph we present the studies of the reduction progress in samples exposed to bending stress. In particular, the influence of this stress on the reduction kinetics was investigated. In the evaluation of reduction degree maps, the effect of a reduction rate enhancement in stressed areas was observed. An example of this effect is clearly visible in the images presented in figure 5, and derived from our analyses of the energy resolved imaging experiments. The maps of reduction degree for two samples, which were reduced together, at the same reduction temperature $\left(700^{\circ} \mathrm{C}\right)$ and atmosphere, are shown. Figure 5a presents a map of a sample reduced under applied bending stress, while the image in figure $5 \mathrm{~b}$ was derived for a reference sample (reduced without external load).

Using the presented quantification algorithm, the reduction degree can be calculated for each pixel resulting in a 2D map. However, better statistical information can be obtained by calculating the average reduction degree for a chosen part of the sample. It is apparent in figure 5 that the reduction reaction was more advanced for the stressed sample (Fig. 
5a) than for reference sample (Fig. 5b). Averaged reduction degrees of the full samples were $33 \%$ and $27 \%$ for the stressed and the reference samples, respectively. A similar effect was observed for samples reduced for 3 and 4 minutes at $700{ }^{\circ} \mathrm{C}$, resulting in significantly higher reduction rate in loaded ( $29 \%$ for $3 \mathrm{~min}$ and $33 \%$ for $4 \mathrm{~min}$ ) then in reference (unloaded) samples (19\% both for $3 \mathrm{~min}$ and $4 \mathrm{~min}$ ). It should be noted that for short reduction times, time measurement error was 1 min due to late response of the setup for atmosphere change.

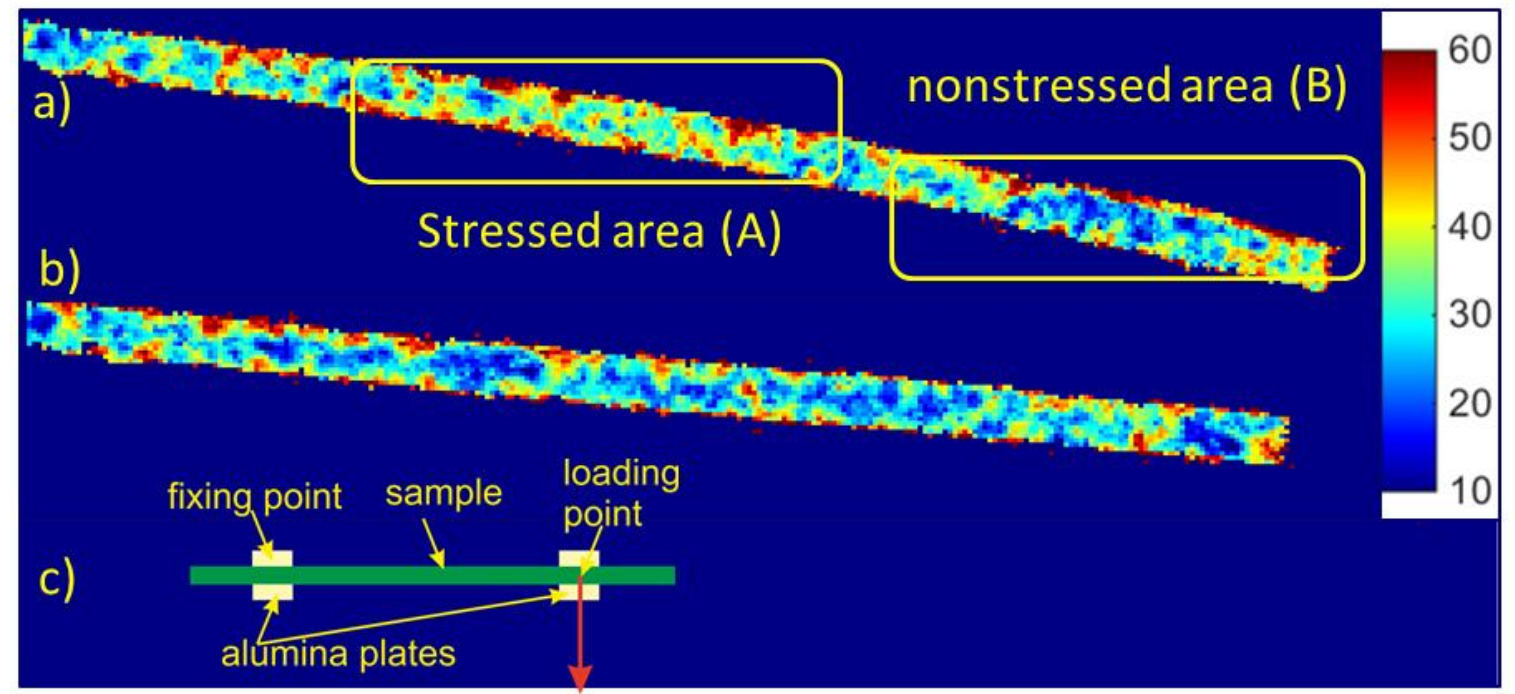

Fig.5 Reduction degree maps for two samples exposed to reducing atmosphere at $700{ }^{\circ} \mathrm{C}$ for 5 minutes: a) sample reduced under applied bending stress, b) reference sample reduced without external load and c) scheme showing the fixing and loading points of the sample.

Moreover, the reduction degrees were extracted for different areas of the bent sample, which are marked in the figure 5a. These two areas correspond to the bent part of the sample (area A), where the highest stress was applied during reduction, and a part of the sample behind the load fixing point, where stresses were at least negligible (area B). The corresponding stress field was determined by the geometry of the fixture applying a load to the sample ${ }^{12}$. The reduction degree calculated for the stressed part A of the sample was $39 \%$ and for the nonstressed part B it was only $28 \%$, corresponding well to the reference sample. These numbers indicate that the reduction reaction was significantly more advanced in the stressed sample compared to the reference sample, and in particular the reduction process was significantly more advanced in the volume effectively exposed to the highest stress.

The corresponding values for a systematic study of samples with different reduction times were plotted as a function of reduction time in figures 6 and 7. Figure 6 presents the derived reduction degrees of samples reduced at $650{ }^{\circ} \mathrm{C}$ evaluated separately for the stressed (figure 6a) and nonstressed parts (figure 6b) of these samples. 

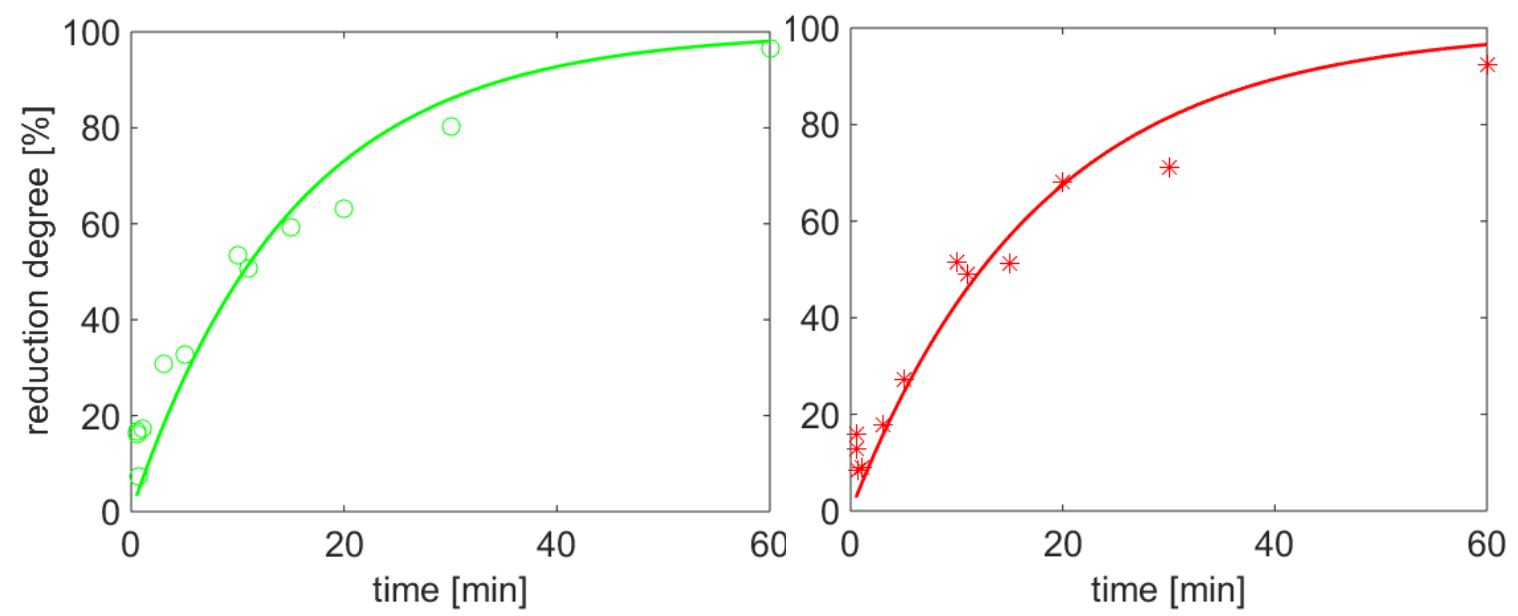

Fig.6 Reduction degrees against reduction time calculated for stressed (green data points) and nonstressed (red data points) parts of sample at $650^{\circ} \mathrm{C}$.

Besides the data points, the presented curves in figure 6 are best fits to the data points with equation 3 . The values achieved for the nonstressed area are better described by this trend, while in the plot for the stressed parts of samples the reduction rates seem to be faster in the beginning than predicted with respect to the fit function, indicating an additional accelerating mechanism at earlier reduction states, which does not allow fitting with the simple equation 3 anymore. Plotting these curves together (fig. 7) clearly shows that reduction rate was enhanced in the stressed parts compared to the nonstressed parts of the samples. Figure 7 includes also results for samples reduced at $700{ }^{\circ} \mathrm{C}$. Enhancement of the reduction rate due to applied stress seems even more significant at higher temperature (at $700{ }^{\circ} \mathrm{C}$ ). Only three samples were measured at $700{ }^{\circ} \mathrm{C}$ for a very short range of time, which does not provide statistics sufficient for indisputable conclusion, but the trend matches the results at $650{ }^{\circ} \mathrm{C}$ well.

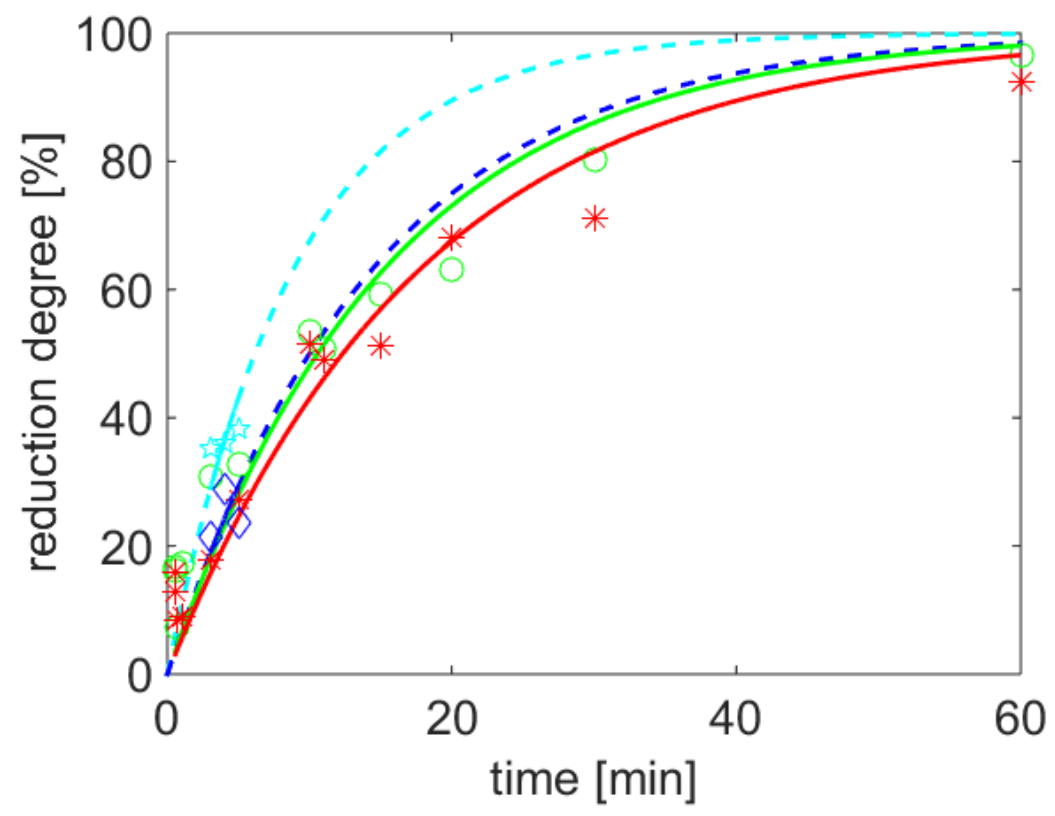

Fig.7 Reduction degrees against reduction time calculated for stressed (green $-650{ }^{\circ} \mathrm{C}$, light blue $-700{ }^{\circ} \mathrm{C}$ ) and nonstressed (red $-650^{\circ} \mathrm{C}$, dark blue $-700^{\circ} \mathrm{C}$ ) parts of samples. 
Effect of compression and tension on reduction process. In order to investigate the effect of compressive and tensile stresses, the stressed part of the sample was divided into three slices, which correspond to compressed (inset in fig. 8a), center (inset in fig. 8b) and tensed side (inset in fig. 8c) of the sample.
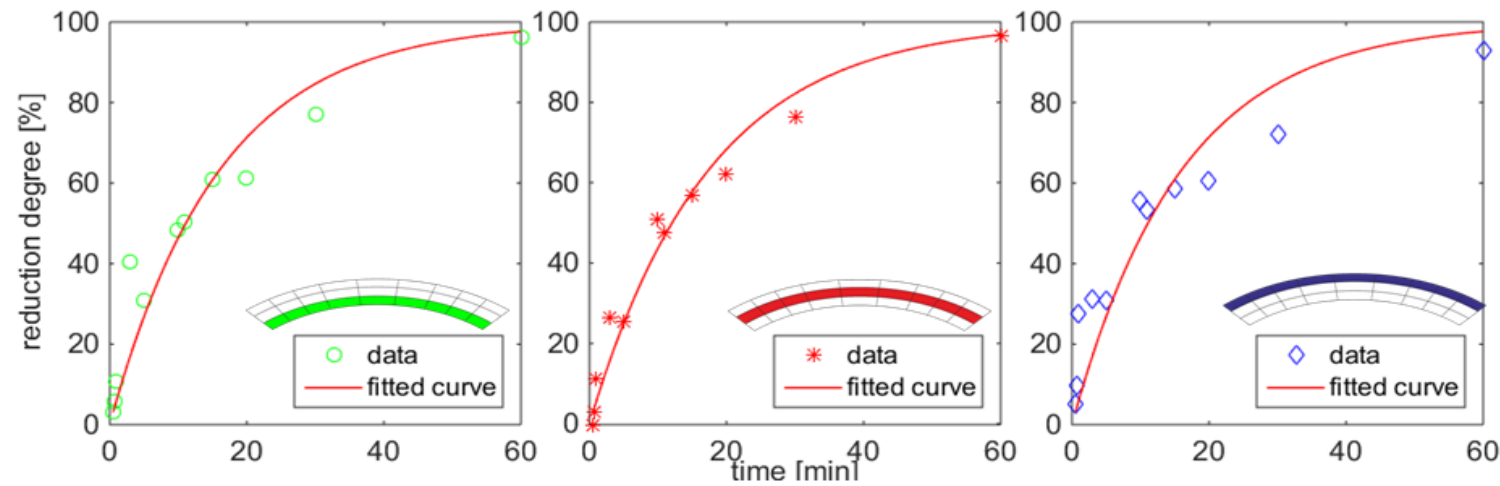

Fig.8 Reduction degrees against reduction time calculated for different slices of the stressed parts of samples reduced at $650{ }^{\circ} \mathrm{C}$ (green - compressed slice, red - center slice, blue - tensed slice). Red lines are curves fitted to the data using the same function from eq. 3 .

The effect of compression and tension is apparent, when compared to the center slice, for which the data has the best agreement with the trend line and where the stress was also the lowest. However, the difference between the effect of compressive and tensile stresses on reduction rate is not so obvious. In both cases, reduction was faster in the first phase of the process compared to the trend line.

The current hypothesis is that compression can nucleate the reduction and enhance the reduction rate especially in the beginning, which was discussed in ${ }^{14}$, but from results presented in fig 8 also tension is seen to enhance the reduction rate.

\section{Conclusion}

In this work the results of energy resolved time of flight (TOF) neutron imaging investigations of the Ni-YSZ cermet used for SOFC anode supports are presented. These experiments were performed at the pulsed neutron spallation source of J-PARC (Japan) using the NOBORU instrument. We present a methodology of analysis of the achieved data and how to extract from these data local information about $\mathrm{Ni}$ and $\mathrm{NiO}$ phases distribution in order to build a map of reduction degree for particular samples.

We have investigated a series of samples reduced at $650^{\circ} \mathrm{C}$ under applied load, as well as few loaded samples reduced together with reference (unloaded) samples at $700^{\circ} \mathrm{C}$. Loaded samples were compared with their reference samples. Moreover, local reduction degrees in parts with different stress concentration within particular samples were studied. Analysis of the achieved information has shown that applying load during reduction has an accelerating influence the reduction rate.

$\mathrm{In}^{14}$ the reduction rate enhancement due to compressive stress was presented. In this work, the effect of compression and tension on reduction rate was investigated as well, however no significant difference was observed and both compression and tension had accelerating influence on reduction rate.

An hypothesis for this could be that bending of the sample due to the accelerated creep on the tensioned side leads to expansion of the layer length, which can result in increase of pore sizes or even opening new pores, therefore once the sample is already 
bent, on this side the gas access is much easier than on the compressed side. On the other side, both compression and tension can simply have the same effect, meaning that stress can enhance the reduction rate, regardless of stress direction. In order to better understand the effect of compression and tension on reduction process, more research, especially in the very early stage of reduction is required. Preferably such research should be performed in-situ, which will be the next step in the studies presented here.

\section{Acknowledgments}

The authors would like to acknowledge DanScatt for financial support and John Johnson for his assistance with sample preparation.

\section{References}

1. S. Singhal; Solid State Ionics 135, 305-313 (2000).

2. J. Malzbender, R. W. Steinbrech, L. Singheiser; Fuel Cells 9, 785-793 (2009).

3. A. B. Stambouli, E. Traversa; Renew. Sustain. Energy Rev. 6, 433-455 (2002).

4. A. Hauch, S. D. Ebbesen, S. H. Jensen, M. Mogensen; J. Electrochem. Soc. 155, B1184 (2008).

5. T. Klemens $\varnothing$, Relationships between structures and performance of SOFC anodes. $\mathrm{PhD}$ Thesis (2005).

6. T. Klemens $\varnothing$, C. C. Appel, M. Mogensen, Lett, E. S. \& A-a, P.; Electrochem. Solid-State Lett. 9, A403-A407 (2006).

7. Y. Wang, M. Walter, K. Sabolsky, M. Seabaugh; Solid State Ionics 177, 15171527 (2006).

8. T. S. Li, W. G. Wang, H. Miao, T. Chen, C. Xu; J. Alloys Compd. 495, 138-143 (2010).

9. P. T. Moseley, A. Hauch, P .S. Jørgensen, K. Brodersen, M. Mogensen; J. Power Sources 196, 8931-8941 (2011).

10. B. Sun, R. A. Rudkin, A. Atkinson; Fuel Cells 9, 805-813 (2009).

11. W. Fischer, J. Malzbender, G. Blass, R. W. Steinbrech; J. Power Sources 150, 73-77 (2005).

12. M. G. Makowska et al.; J. Appl. Crystallogr. 48, 401-408 (2015).

13. S. B. Simonsen et al.; Appl. Catal. A Gen. 489, 147-154 (2015).

14. H. L. Frandsen et al.; Conf. Proc. Eur. Fuel Cell Forum, Lucerne, Switz. (2014).

15. K. Iwase, K. Sakuma, T. Kamiyama, Y. Kiyanagi; Nucl. Instruments Methods Phys. Res. Sect. A. 605, 1-4 (2009).

16. A. S. Tremsin et al.; Strain 48, 296-305 (2012).

17. R. Woracek et al.; J. Appl. Phys. 109, 093506 (2011).

18. J. R. Santisteban, L. Edwards, V. Stelmukh ; Phys. B Condens. Matter 385-386, 636-638 (2006).

19. J. R. Santisteban, L. Edwards, M. E. Fizpatrick, A. Steuwer, P. J. Withers; 1436, 1433-1436 (2002).

20. L. Josic, E. Lehmann, A. Kaestner; Nucl. Instruments Methods Phys. Res. Sect. A. 651, 166-170 (2011).

21. N. Kardjilov et al.; Int. J. Mater. Res. (formerly Zeitschrift fuer Met. 103, 151154 (2012).

22. E. Lehmann, A. Kaestner, L. Josic, S. Hartmann, D. Mannes; Nucl. Instruments Methods Phys. Res. Sect. A. 651, 161-165 (2011). 
23. S. Vogel, A Rietveld-Approach for the Analysis of Neutron Time-Of-Flight Transmission Data.; PhD Thesis (2000).

24. Woracek, R. et al.; Adv. Mater. 26, 4069-73 (2014).

25. W. Treimer, M. Strobl, N. Kardjilov, A. Hilger, I. Manke; Appl. Phys. Lett. 89, 203504 (2006).

26. M. Strobl et al.; Nucl. Instruments Methods Phys. Res. Sect. A 651, 149-155 (2011).

27. M. Strobl et al.; Nucl. Instruments Methods Phys. Res. Sect. A. 680, 27-34 (2012). 28. J.R. Santisteban, L. Edwards, A. Steuwer, P.J. Withers ; J. Appl. Crystallogr. 34, 289-297 (2001).

29. A.S. Tremsin et al.; Nucl. Instruments Methods Phys. Res. Sect. A 633, S235S238 (2011).

30. F. Maekawa et al.; Nucl. Instruments Methods Phys. Res. Sect. 600, 335-337 (2009).

31. K. Oikawa et al.; Nucl. Instruments Methods Phys. Res. Sect. A. 589, 310-317 (2008).

32. A. S. Tremsin et al.; Nucl. Instruments Methods Phys. Res. Sect. A 688, 32-40 (2012).

33. A. S. Tremsin, J. V. Vallerga, J. B. McPhate, O. H. W. Siegmund, R. Raffanti; IEEE Trans. Nucl. Sci. 60, 578-585 (2013).

34. S. Biswas, T. Nithyanantham, N. T. Saraswathi, S. Bandopadhyay; J. Mater. Sci. 44, 778-785 (2008).

35. M. Radovic et al.; Ceram. Eng. Sci. Proc. 281-286 (2004). 